Nutr. Metabol. 1975;19:111-112

\title{
Book Reviews · Buchbesprechungen · Livres nouveaux
}

Ergebnisse der Experimentellen Medizin, vol. 17: Neurobiological Basis of Memory Formation, part II. VEB Verlag Volk und Gesundheit, Berlin. 279 pp.

This book of 279 pages is bound in paperback. It contains 17 contributions all in English and the majority of them well-illustrated with tables and diagrams. With the exception of the United States (5) and the Netherlands (2) the contributions are from Eastern Germany, Czechoslovakia and Russia. All the contributions have worthwhile bibliographies and those from Russia may therefore be particularly useful. The material presented includes the following: neuroglia and memory; biochemical correlates of carbon dioxide-induced amnesia in rats; effect of early postnatal malnutrition and pharmacological treatment on behaviour; learning and biochemical variables in adult rats; pharmacologic manipulation of memory processing in the neonate chick model; brain stimulation and the production of retrograde amnesia; genetic and biochemical analysis of individual differences in learning; are the cortical proteins involved in the visual discrimination training?

We are still far from understanding the memory process, and investigators of the subject, from whatever point of view, will find something in this book to interest them. Those who do have the book, however, will first want to prepare a 'Table of Contents' and, possibly, an 'Index', for it lacks both.

/. W. T. Dickerson, Guildford

P. Kopp und K. Trautner (Hrsg.): Entwicklungstendenzen in Ernährung und Diätetik. Bibliotheca Nutritio et Dieta, No. 21. Karger, Basel 1975. VIII + 205 pp., 42 fig., 29 tab.; SFr. 97.-/DM 92.-/ approx. US \$ 44.25. ISBN 3-8055-1737-8.

Eine schönere Ehrung kann man sich zu einem „runden Geburtstag” kaum wünschen als die, die dem Leiter des Institutes für Ernährungsforschung, Rüschlikon, Zurich, Prof. Dr. J.C. Somogy widerfuhr: Ein Symposium über die Entwicklungstendenzen des von ihm ver-tretenen Faches. Die schweizerische Ernährungsforschung und nicht zuletzt der Jubilar selbst haben, wie K. Bernhard in seinem Einleitungsreferat ausführt, viel zur Entwicklung des Wissens auf diesem Gebiet und für seine Anwendung in der Praxis getan. Die Thematik der Vorträge waren - dem umfassenden Titel entsprechend - sehr vielseitig: allgemeines über Ernährung und Diätetik; Eiweiss, einschliesslich neuer unkonventioneller Quellen; Fet-te; Zucker; Makro- und Mikroelemente, und von den Vitaminen die Ascorbinsäure waren die Diskussionsgegenstände. Der Internationale Kreis von Referenten und Diskutanten garantier-te das Niveau der Veranstaltung, deren Verhandlungen hiermit in Buchform erscheinen.

H.-D. Cremer, Giessen

H.D. Cremer und D. Hötzel (Hrsg): Ernährungslehre und Diätetik. Ein Handbuch in 4 Bänden, vol. 3: Angewandte Ernährungslehre. Thieme, Stuttgart 1974. XV + 386 pp., 45 fig., 136 tab.; DM 198.--

Wie die Herausgeber im Vorwort bemerken, wurde erstmals 1897 von E. von L·eyden im Georg Thieme Verlag Leipzig ein „Handbuch der Ernährungstherapie und Diätetik” herausgegeben und in 2. Auflage 1903 verlegt. Das heutige vierbändige Werk entspricht 
Book Reviews $\mathbf{~ B u c h b e s p r e c h u n g e n ~ · ~ L i v r e s ~ n o u v e a u x ~} 112$

offenbar einer späten Neuauflage, dürfte jedoch ausser dem Verlag nicht mehr viel Gemeinsames mit dem Leydenschen Handbuch aufweisen. Es fällt wohl nicht zufällig in eine Zeit, da der Ernährung nicht nur in politischen Gremien, sondern auch in der Klinik aktuellste Bedeutung zukommt.

Unter dem Titel „Angewandte Ernährungslehre” enthält Band 3 ein breites Spektrum von Beiträgen, verfasst von Ernährungsphysiologen, Medizinern, Lebensmittelchemikern, Soziologen und Ernährungsberatern. Es sind teils Themen von ganz allgemeinem Interesse wie die Problematik der Ernährung der Weltbevölkerung (Wirths und Cremer), die Nahrungsversorgung der Bevölkerung am Beispiel der Bundesrepublik Deutschland (Wirths) oder Verzehrsgewohnheiten (Pfefferj. Methoden zur Ermittlung des Ernährungszustandes (Wirths) interessieren wohl mehr Kliniker, Epidemiologen und Diätassistentinnen, ebenso was man unter vollwertiger Ernährungg des Gesunden (Wirths) versteht. Eine Einfúhrung in die Lebensmittelchemie und das Lebensmittelrecht (Bitsch und Bitsch) richtet sich zweifellos mehr an Chemiker und Beamte der öffentlichen Gesundheitsdienste. Rund 150 von 380 Seiten sind der Lebensmitteltechnologie (diverse Autoren) gewidmet. einem weiten Gebiet, das Lebensmittelchemiker, Technologen, aber auch Diätassistentinnen und schliesslich Kliniker zu interessieren vermag. Gesamthaft liegt hier ein modernes interdisziplinäres Werk vor, ausgestattet mit zahlreichen, jedoch ausgewählten Literaturangaben neueren Datums, dem eine weite Verbreitung bei alien an Ernährungsproblemen Interessierten zu wünschen ist.

G. Hartmann, Chur 